\title{
Questes
}

vestes Revue pluridisciplinaire d'études médiévales

La règle du jeu

\section{Réguler la pratique théâtrale}

\section{Simon Gabay}

\section{(2) OpenEdition \\ Journals}

Édition électronique

URL : http://journals.openedition.org/questes/661

DOI : 10.4000/questes.661

ISSN : 2109-9472

\section{Éditeur}

Les Amis de Questes

\section{Édition imprimée}

Date de publication : 15 février 2010

Pagination : 44-53

ISSN : 2102-7188

\section{Référence électronique}

Simon Gabay, «Réguler la pratique théâtrale », Questes [En ligne], 18 | 2010, mis en ligne le 03

septembre 2015, consulté le 20 avril 2019. URL : http://journals.openedition.org/questes/661 ; DOI :

$10.4000 /$ questes.661 


\title{
Réguler la pratique théâtrale
}

\author{
Simon GABAY
}

Sans entrer dans des considérations complexes de genre, il peut paraître utile de séparer un instant le théâtre du reste de la littérature, pour mettre en avant sa principale caractéristique. Le genre dramatique se distingue en effet des autres par un fait important: il ne se réduit pas uniquement au texte, mais se matérialise sous une forme concrète lors de sa représentation ${ }^{1}$. Il existe donc grosso modo deux théâtres : l'un écrit et donc immuable, l'autre joué et par nature éphémère. La question du contexte, même si elle se pose pour les autres genres littéraires ${ }^{2}$, prend donc ici une autre envergure, car si l'écriture est dans une certaine mesure dégagée des conditions de production, la représentation ne l'est pas.

Deux postures théoriques, dont les résultats se situent sûrement sur le continuum tracé par ces deux extrêmes, s'offrent alors au chercheur. D'une part, il peut se concentrer sur la nature littéraire de la pièce et donc sur sa dimension textuelle; d'autre part, il peut opter pour une insistance particulière sur son aspect spectaculaire, considérant la représentation comme l'objectif final de l'existence même du théâtre. Nous souhaitons nous tourner vers la seconde option. Qui joue ? Comment? Quand ? Où? Pour qui ? Pourquoi? Il est donc ici question de nous intéresser à une histoire particulière, celle du théâtre joué.

La période médiévale se prête particulièrement à cette analyse « historique » de la littérature, car contrairement à d'autres époques, peu de

\footnotetext{
${ }^{1}$ Nous n'impliquons pas ici un enchaînement logique des deux temps théâtraux que sont l'écriture et le jeu: le texte peut être modifié (adaptation au public, trous de mémoire...), ne pas être joué (le «théâtre dans un fauteuil» de Hroswitha de Gandersheim et d'autres, bien que cette idée soit discutée) ou ne pas connaître de version écrite (improvisation théâtrale).

${ }^{2}$ Nous pensons bien évidemment au débat tournant autour de l'oralité de la littérature médiévale.
} 
pièces nous sont parvenues : il faut donc retrouver celles qui survivent dans les archives, ne serait-ce que par de simples références. Les documents liés à la pratique théâtrale sont ainsi à la fois l'occasion de s'intéresser à la dimension concrète du théâtre, qui est encore trop mal connue, mais aussi de tenter de combler le manque de matière première "littéraire ». Si nous partons de la pratique, ce n'est donc aucunement pour nous y cantonner, mais plutôt pour donner un point de départ qui, nous l'espérons, aidera à s'interroger de manière plus globale sur la nature du théâtre français du Moyen Âge.

Nous nous proposons ici d'expliciter quelque peu cette méthode et de donner un aperçu de nos recherches pour partager notre démarche et nos doutes.

\section{Pratique théâtrale et archives}

Nous nous proposons de fournir des réponses aux questions posées ci-dessus via les archives juridiques. Il va de soi que les archives sont une source d'informations indépassable pour la vie de la société médiévale, et dès lors que l'art théâtral sort de son texte pour rejoindre la scène, des traces sont gardées dans les annales des lieux de pouvoirs. Il s'agit donc de retrouver les mentions relatives à notre sujet, mais le corpus étant évidemment bien trop vaste, il a fallu d'abord quadriller puis restreindre le lieu de fouille, et c'est le corpus juridique qui a été retenu.

L'archive juridique possède en effet un double avantage : elle donne des faits et porte une appréciation sur eux. Nous avons ainsi dans une unique source des informations brutes, accompagnées d'une vision de ces informations par ses contemporains. Nous faisons d'une pierre deux coups : nous reconstruisons à la fois la pratique théâtrale du Moyen Âge et, via les jugements, la vision de cette pratique. Plus concrètement, nous avons conservé des traces des jugements médiévaux, et nous y trouvons parfois 
sur le banc des accusés les acteurs du théâtre médiéval, les contextes et les motifs d'inculpation qui nous sont nécessaires pour reconstruire patiemment ce que peut être une pièce du Moyen Âge lorsqu'elle est jouée.

Cette méthode a pour but de questionner des idées universellement admises pour ce qui relève du théâtre, de différencier, sans les séparer, l'écrit et la pratique, et enfin de dégager les spécificités de l'époque médiévale. Règne encore trop souvent dans les esprits l'image de l'acteur banni de la société et poursuivi par l'Église, jusqu'à son enterrement sans prêtre aux portes de la ville - autrement dit l'image floue de Molière. Cette conception de l'Église catholique (puis des diverses branches du protestantisme dans ses foyers les plus importants sur le sol français) vivant dans la haine du théâtre et suivant à la lettre les préceptes de vie énoncés par l'évêque d'Hippone ${ }^{3}$ est-elle vraie ? Comment comprendre alors le rôle fréquent de commanditaire qu'ont joué les autorités ecclésiastiques?

\section{Les archives ecclésiastiques}

Il faut donc se concentrer sur un type précis d'archive juridique : les archives ecclésiastiques, qui s'opposent à celles du pouvoir séculier. L'importance de l'Église au Moyen Âge ne peut en effet qu'inciter à mieux comprendre quelle fut son attitude vis-à-vis du théâtre, et à tenter de saisir plus correctement le paradoxe entre la théorie (contre) et la pratique (pour) ${ }^{4}$. La concentration sur les questions du droit canonique et des

\footnotetext{
${ }^{3}$ Par exemple dans Les Confessions. Pour plus de précisions, cf. Jean-Baptiste ÉRIAU, Pourquoi les Pères de l'Église ont condamné le théâtre en leur temps?, Paris, Champion, 1914.

${ }^{4}$ Nous ne sommes bien entendu pas les premiers à nous intéresser au problème, et l'on trouvera dans les classiques que sont les livres d'Alessandro D'AnCONA, Origini del Teatro in Italia, Florence, Successori Le Monnier, 1877, Edmund ChAmBERS, The Medieval Stage, Oxford, Oxford University Press, 1978 (1903) ou Karl Young, The Drama of the Medieval Church, Oxford, Clarendon Press, 1967 (1933) de multiples pistes. Tous ont cependant relevé le paradoxe sans tenter véritablement de le résoudre (notamment Karl Young, The Drama of the Medieval Church, op. cit., t. II, p. 410421).
} 
positions des courants théologiques ne donne qu'une idée partielle du problème : nécessaire, cette première étude doit désormais se doubler d'une seconde mesurant l'impact de ces pensées normatives sur le quotidien.

L'Église est hiérarchiquement organisée, du Pape au simple fidèle, avec entre ces deux extrêmes une multitude de degrés du sacrement de l'ordre (diacre, prêtre, évêque...). Suivant ce degré, plusieurs fonctions peuvent être exercées (le prêtre peut être curé, vicaire ou chanoine), et à ces fonctions peuvent être adjointes certaines dignités (le chanoine peut-être écôlatre, ecclésiarque, chantre, official...). Des jugements émanent des différents niveaux de cette organisation (bulle, concile, synode, jugement d'officialité...) et s'ils n'ont pas la même valeur juridique, ils participent tous d'une même mentalité ; il s'agit de les analyser ensemble, tout en gardant à l'esprit leur nature différente, pour saisir pleinement la vision du théâtre par l'Église.

La voix de l'official est ainsi un choix logique pour mettre en pratique notre idée, son rôle étant de rendre cette justice ecclésiastique au nom de l'évêque dans le diocèse - les délibérations capitulaires sont une autre possibilité 5 . Il a pour cela un personnel, des locaux, un code et une procédure à suivre, et c'est dans ce cadre qu'il juge les cas liés au théâtre. ${ }^{6}$ Les jugements qui nous intéressent se trouvent essentiellement dans les archives criminelles (nous dirions aujourd'hui pénales) et non civiles, ces dernières concernant les rapports entre individus, non pas des comportements considérés comme antisociaux. Nous trouverions sûrement des documents relatifs au théâtre dans des cas de jugements au civil, mais

\footnotetext{
${ }^{5}$ Elles ne sont bien évidemment pas proprement juridiques, relevant de la gestion plus que du jugement, mais les choix opérés par les chanoines impliquent des autorisations et des oppositions, tombant donc dans notre champ d'étude défini de manière large. Évidemment, nous y reviendrons à l'avenir.

6 Deux livres essentiels sont consacrés à la question de l'official, celui de Paul Fournier, Les Officialités au Moyen Âge, Paris, Plon, 1880, et celui d'Anne LeFEBVReTeILlard, Les Officialités à la veille du Concile de Trente, Paris, LGDJ, 1973.
} 
ils seraient relatifs à des problèmes de contrat entre des gens de théâtre, et non des problèmes de théâtre entre l'Église et diverses parties. Dans l'idée de chercher une vision du théâtre médiéval « en creux », il nous paraît donc moins important, bien que par ailleurs tout à fait légitime, de nous intéresser aux poursuites au civil ${ }^{7}$. En jugeant ce qui est antisocial, le jugement criminel nous donne les limites posées à la pratique théâtrale, et en multipliant les exemples nous espérons atteindre un aperçu de la vision de cette pratique par ses contemporains.

\section{Cas pratique}

Les documents que nous avons sont dans une grande partie conservés dans les registres des causes criminelles, les pièces de procédures, les registres d'audience ou les registres de compte appartenant aux séries $G$ des archives départementales et de la série $Z$ des archives nationales (qui contiennent les archives de Paris) sous la dénomination « officialité ». Ils s'organisent souvent d'une manière simple, avec le nom, la profession, l'adresse, le motif d'inculpation, le jugement et l'amende. Nous proposons d'étudier un acte se trouvant à la référence $\mathrm{Z1}^{\circ}-18$, $\mathrm{f}^{\circ} 189 \mathrm{r}^{\circ}, \S 5^{8}$.

«Gaspardus Famart stagnarius gallice potier d'estain commorans in loco cimeteri sancti Johannis etc. emendavit lusisse certum ministerium seu ludum de vita sancti mathurini in quadam aula absque hoc quod ludus fuisset visitatus taxata ad iiij solidos et dimendium salarius. »

(Gaspard Famart, potier d'étain, demeurant dans le quartier du cimetière Saint-Jean, etc. est condamné à quatre sous parisis et à

\footnotetext{
${ }^{7}$ Notre propre spécialisation n'est ainsi pas arbitraire : elle se place dans le cadre d'un projet global, où la législation civile sera étudiée par d'autres. Marie BOUHAÏK-GIRONES et Katell LAVEANT travaillent ainsi actuellement sur le problème du droit civil, cf. « $\mathrm{S}$ 'associer pour jouer. Actes notariés et pratique théâtrale, $\mathrm{XV}^{\mathrm{e}}-\mathrm{XVI}^{\mathrm{e}}$ siècles », L'Acteur et l'accessoire. Mélanges en l'honneur de Michel Rousse, à paraître.

${ }^{8}$ Cet exemple est tiré de la thèse de Léon POMMERAY, L'Officialité archidiaconale de Paris, Paris, Sirey, 1933, n. 1 p. 420, ainsi que quelques-unes des conclusions qui suivent.
} 
un demi-salaire pour avoir joué un certain mystère ou jeu de la vie de saint Mathurin dans une certaine cour sans que ce jeu soit approuvé.)

C'est ici un jugement de l'officialité archidiaconale de Paris du 18 septembre 1486. Nous y voyons tous les éléments dont nous avons parlé : le nom («Gaspardus Famart»), le métier («stagnarius gallice», dont il est intéressant de noter la traduction en français juxta, «potier d'estain », à une époque où le latin est toujours la langue de l'État, mais où le français commence à s'affirmer de plus en plus), l'adresse (« commorans in loco cimeteri sancti Johannis»), le motif d'inculpation ("emendavit lusisse certum ministerium seu ludum de vita sancti mathurini in quadam aula absque hoc quod ludus fuisset visitatus ») et la sentence («taxata ad iiij solidos et dimendium salarius »).

Plusieurs points méritent d'attirer notre attention. Tout d'abord, le condamné n'est pas un clerc, ce qui nous permet de dire que l'officialité juge encore à cette époque des laïcs (de condition basse ${ }^{9}$ ), et que des laïcs sont mêlés à des représentation de pièces pastorales. Ensuite, la terminologie reste flottante («certum ministerium seu ludum »), et le juge, pourtant linguistiquement incité à la précision, n'est pas plus apte à définir l'objet théâtral que ses concitoyens ${ }^{10}$. Enfin, il convient de remarquer l'essentiel : la pièce n'a pas été autorisée par les autorités, ce qui sousentend l'existence d'un organe de censure, donc bien avant la Réforme ${ }^{11}$.

Élargissons maintenant la portée du cas à l'aide d'autres textes, en espérant donner de la profondeur au nôtre par une insertion dans son

\footnotetext{
${ }^{9}$ Plus précisément, une personne n'appartenant ni à l'aristocratie, ni à la bourgeoisie, mais tout de même pas en marge de la société, comme les clercs vagants, (cf. Helen WADDELL, The Wandering Scholars, Londres, Constable, 1927).

${ }^{10}$ Il s'agit cependant de se méfier de l'emploi de ces doublons que nous trouvons souvent dans les archives et qui sont le fait d'un style juridique.

${ }^{11}$ Sur les problèmes du théâtre, de la Réforme et du droit, on pourra se référer, entre autres, à l'article de Katell LAVEANT, « Le théâtre du Nord et la Réforme », European Medieval Drama, 11 (2007), p. 59-77.
} 
contexte. Regardons pour cela trois types de documents qui peuvent nous être utiles : premièrement les autres cas du registre, deuxièmement les cas d'autres régions, troisièmement et dernièrement les documents émanant d'instances hiérarchiquement plus élevées dans l'organigramme ecclésiastique.

Si nous intégrons ce document dans le registre d'où il est tiré, nous pouvons dire que c'est là un des derniers cas de jugement d'un laïc par l'official, le dernier intervenant le 4 octobre $1486^{12}$. Cette situation n'est pas claire, mais peut-être que les conflits de juridiction peuvent expliquer cette situation $^{13}$. Cela nous amène à un second point : la rareté des cas relatifs au théâtre dans les registres de Paris amène à relativiser l'importance du phénomène théâtral du point de vue juridique. L'acharnement théologique et législatif n'a pas de conséquences proportionnelles dans la pratique juridique, ce qui pourrait impliquer une relative distance vis-à-vis du code, ou alors, plus intéressant, une erreur de compréhension de celui-ci par les exégètes modernes ${ }^{14}$.

Élargissons maintenant un peu plus le champ des documents utilisables, en faisant appel à ceux d'autres lieux. Il est fait mention de l'absence d'autorisation dans le cas de Gaspard Famart, sous-entendant l'existence d'un organisme de censure dont il serait intéressant de comprendre un peu mieux le mécanisme. Grâce à l'arrêt du 21 juillet 1488 («habuissent licentiam seu permissionem a domino sigillifero »), nous pouvons savoir qu'à Paris l'organe de censure passe très probablement par

\footnotetext{
12 Information relevée par Léon POMMERAY, L'Officialité archidiaconale de Paris, op. cit., p. 420, corroborée par nos sondages dans les registres de l'officialité.

${ }^{13}$ Les acteurs, mêmes clercs, passant dans plusieurs cas aux mains de la justice séculière, cf. Marie BouHAÏK-GIRONES, Les Clercs de la Basoche et le théâtre comique, Paris, Champion, 2007.

${ }^{14}$ Karl Young expose cette possibilité dans The Drama of the Medieval Church, op. cit., t. II, p. 416, en citant une glose d'une décrétale d'Innocent III : les condamnations du théâtre par la hiérarchie ne concernent pas sa nature même, mais des formes précises qu'il prendrait.
} 
le scelleur de l'officialité archidiaconale. Mais d'autres cas se présentent: une mention des délibérations capitulaires du chapitre métropolitain de Saint-André de Bordeaux (G. 286, fo $315^{15}$ ) nous explique que le chapitre reçoit des demandes d'autorisation (en l'occurrence une pièce comique que des enfants veulent jouer), auquel il répond en transmettant la relecture à un membre externe à l'officialité (le sous-doyen) : doit-on imputer cette différence de fonctionnement à des pratiques différentes, ou au fait que la pièce provienne d'un milieu scolaire? Une étude plus poussée de la censure ecclésiastique, que nous menons actuellement, pourrait permettre d'y répondre. Notons, en prolongement de la première remarque, que le problème de l'autorisation n'apparaîtra plus après le 19 septembre 1517 dans les registres de l'officialité archidiaconale de Paris, et qu'il convient aussi de s'interroger sur les raisons de cette disparition.

Finissons par un troisième niveau de lecture, non plus diatopique ou diachronique, mais diastratique en regardant vers le haut de la hiérarchie. Nous pouvons rapprocher ce jugement des décisions du récent concile de Sens (qui concerne Paris), tenu un an avant la condamnation de Gaspard, exigeant une autorisation (cette fois de l'Ordinaire) pour jouer $^{16}$. Il est donc extrêmement clair que l'Église ne condamne pas le théâtre, mais un théâtre : contrôler, c'est accepter, ou tout du moins tolérer; la nature même de l'art dramatique n'est plus source de réprobation. Il apparaît aussi à la lumière de ces décisions que l'excommunication du comédien, sans dire qu'elle n'est pas pratiquée, n'est pas nécessairement la règle : le fait même que Gaspard soit jugé par un tribunal ecclésiastique prouve qu'il n'est pas excommunié (le tribunal ecclésiastique se trouverait alors incompétent).

\footnotetext{
${ }^{15}$ Toute mention de document se trouvant ailleurs qu'à Paris trouve sa source dans les inventaires des archives départementales concernées.

16 Les représentations doivent se faire «ex speciali permissione Ordinarii, et beneplacito ministrorum ipsius ecclesia », MANSI, Concilia, t. XXXII, col. 413.
} 


\section{Premières conclusions}

Il est maintenant temps de tirer les premières conclusions des quelques cas que nous avons trouvés et des connaissances, toujours trop superficielles, que nous avons acquises. Tout d'abord nous pouvons retrouver, par ces archives juridiques, les traces d'un certain nombre de pièces : la Vie de saint Mathurin $\left(\mathrm{Z1}^{\circ}-18, \mathrm{f}^{\circ} 189 \mathrm{r}\right.$, §5), Miracle et vie de sainte Marguerite de Poitiers (Paris, $\mathrm{Z1}^{\circ}$-18, $\mathrm{f}^{\circ} 192 \mathrm{v}, \S 1$ ), le Mystère sur le Jugement dernier (Paris, $\mathrm{Z1}^{\circ}-19, \mathrm{f}^{\circ} 70 \mathrm{v}, \S 1$ ), le Jeu dit «de l'Enfant Prodigue » (Paris, $\mathrm{Z1}^{\circ}$-22, $\mathrm{f}^{\circ} 187$ r, §2), le Mystère de saint Victor (Rouen, G 338). Les antiquaires du XIX ${ }^{\mathrm{e}}$ siècle et les universitaires ayant exploité et approfondi leur travail ${ }^{17}$ sont nombreux, mais la documentation n'est pas encore épuisée, ou bien nécessite parfois d'être remise en valeur. Le cas d'une farse écrite (et jouée) contre Robert Charpentier par un ecclésiastique d'Aconville (Rouen, G 357), menant à la condamnation des deux hommes par l'Official, peut ainsi nous permettre de faire progresser le répertoire du théâtre médiéval tout en traitant notre problème des rapports entre Église et théâtre.

Des informations importantes nous sont données dans une multitude de cas «satellites » trouvés dans les archives: problème de la tenue vestimentaire, de la fête des fous, de la danse, de la musique... Il est important de ne pas éluder des cas à première vue annexes et finalement connexes au problème qui nous est posé, surtout que le matériau relatif au théâtre n'est pas si abondant. Ce qui amène un juge à condamner un prêtre pour avoir dansé en public ou s'être déguisé éclaire nécessairement notre vision des choses, et il conviendra de nous interroger plus en profondeur

17 De Louis Petit DE Julleville (Les Mystères, t. II, Paris, Hachette, 1880, notamment) à Graham RunNalls (Les Mystères dans les provinces françaises, Paris, Champion, 2004), la liste est longue. 
sur les rapports entre ces différents arts dans la pratique juridique ${ }^{18}$. Ces cas soulèvent ainsi plus nettement que ceux relatifs au théâtre un point important, à Paris tout du moins, et évident : le statut de clerc implique une différence de traitement, et ils se retrouveront plus souvent que les laïcs, qui pourtant jouaient parfois avec eux, devant les juges pour avoir pris part à ces pratiques.

Enfin, notons que tous ces cas sont intéressants en tant que jugements, car ils montrent que, malgré les interdictions, les médiévaux n'obéissent pas aveuglément ; cependant, dire qu'ils font fi de la loi est tout aussi faux. Les rapports du théâtre et de l'Église sont complexes, et les réduire à des condamnations aussi violentes qu'éternelles est autant erroné que de se contenter de constater une schizophrénie ecclésiastique. Il existe des points précis qui délimitent la pratique théâtrale, qui varient selon les lieux (une église ou une rue), les hommes (clercs, ayant ou non une charge sacerdotale, ou laïcs), le temps (jour de semaine ou jour de fête) : ce sont ces frontières qu'il faut chercher à mettre en évidence et à comprendre pour trouver une réponse plus fiable.

${ }^{18}$ La notion de performance traduit bien cette similarité, et donc l'impossibilité d'en rester à une définition trop réductrice du «théâtre ». Nous n'avons cependant pas abordé la question des définitions dans cet article, celle-ci ayant connu (et connaissant toujours) d'amples débats. 